Keywords: interleukin-6 (IL-6); resistance; radiation; nuclear factor erythroid 2-related factor 2 (Nrf2); reactive oxygen species (ROS); oral squamous cell carcinoma (OSCC)

\title{
IL-6 controls resistance to radiation by suppressing oxidative stress via the Nrf2-antioxidant pathway in oral squamous cell carcinoma
}

Yuichiro Matsuoka ${ }^{1,7}$, Hideki Nakayama ${ }^{*}, 1,7$, Ryoji Yoshida ${ }^{1}$, Akiyuki Hirosue ${ }^{1}$, Masashi Nagata ${ }^{1}$, Takuya Tanaka ${ }^{1}$, Kenta Kawahara ${ }^{1}$, Junki Sakata ${ }^{1}$, Hidetaka Arita ${ }^{1}$, Hikaru Nakashima ${ }^{1}$, Satoru Shinriki ${ }^{2,3}$, Daiki Fukuma ${ }^{1}$, Hidenao Ogi ${ }^{1}$, Akimitsu Hiraki ${ }^{4}$, Masanori Shinohara ${ }^{1}$, Ryo Toya ${ }^{5}$ and Ryuji Murakami ${ }^{6}$

${ }^{1}$ Department of Oral and Maxillofacial Surgery, Faculty of Life Sciences, Kumamoto University, Kumamoto 860-8556, Japan; ${ }^{2}$ Department of Molecular Laboratory Medicine, Graduate School of Medical Sciences, Kumamoto University, Kumamoto 860-8556, Japan; ${ }^{3}$ Central Clinical Laboratory, Kumamoto University Hospital, Kumamoto 860-8556, Japan; ${ }^{4}$ Section of Oral Oncology, Department of Oral and Maxillofacial Surgery, Fukuoka Dental College, Fukuoka 814-0193, Japan; ${ }^{5}$ Department of Radiation Oncology, Kumamoto University Hospital, Kumamoto 860-8556, Japan and ${ }^{6}$ Department of Medical Imaging, Faculty of Life Sciences, Kumamoto University, Kumamoto 862-0976, Japan

Background: In promoting tumour malignancy IL-6 signalling is considered to have an important role. However, the biological roles of IL-6 on radiosensitivity in oral squamous cell carcinoma (OSCC) remain largely unclear. The objective of this study is to determine the effects and molecular mechanisms of IL-6 on radiosensitivity in OSCC.

Methods: Two OSCC cell lines, and OSCC tissue samples with radioresistant cells were used. We examined the effects of IL-6, or tocilizumab, a humanised anti-human IL-6 receptor antibody, or both on radiosensitivity and DNA damage after X-ray irradiation in vitro. In addition, we investigated the involvement of the Nrf2-antioxidant pathway in IL-6-mediated radioresistant mechanisms using OSCC cell lines and tissues.

Results: Increased levels of IL-6 suppressed radiation-induced cell death, and the blockade of IL-6 signalling by tocilizumab sensitised tumour cells to radiation. The radioresistant effect of IL-6 was associated with decreased DNA damage after radiation. We also found that IL-6 promotes the activation of not only the downstream molecule STAT3 but also the Nrf2-antioxidant pathway, leading to a significant decrease in oxidative stress by upregulating Mn-SOD.

Conclusions: These results indicate that the blockade of IL-6 signalling combined with conventional radiotherapy could augment the treatment response and survival rate in patients with radioresistant OSCC.

Radiotherapy is an important treatment modality in head and neck cancer, including oral squamous cell carcinoma (OSCC), effectively killing cancer cells by causing DNA damage through the generation of reactive oxygen species (ROS) (Zhou et al, 2013).
However, cancer cells with an antioxidant ability can escape the damaging effects of radiation by scavenging ROS, thus leading to radioresistance (Diehn et al, 2009). This radioresistance continues to be a major clinical obstacle to the cure of OSCC.

\footnotetext{
*Correspondence: Professor H Nakayama; E-mail: hinakaya@kumamoto-u.ac.jp

${ }^{7}$ These authors contributed equally to this work.
}

Received 26 May 2016; revised 12 September 2016; accepted 14 September 2016; published online 13 October 2016

(c) 2016 Cancer Research UK. All rights reserved 0007-0920/16 
Exposure to clinically relevant doses of radiation is known to not only induce DNA damage but also activate a large network of inflammatory signalling pathways (Deorukhkar and Krishnan, 2010). In addition, radiation-induced inflammation has a pivotal role in inducible radioresistance for cancer cells (Multhoff and Radons, 2012). Therefore, if we can interrupt the inflammatory networks by targeting the inflammatory key molecule, we may offer a novel therapeutic approach to improve the clinical outcome of radiotherapy by enhancing radiosensitivity. However, the details regarding the molecular mechanism of inflammation-induced radioresistance remain unclear in OSCC.

Interleukin-6 is a multifunctional cytokine that was originally determined to regulate immune and inflammatory responses and inhibits apoptosis in toxic environments during inflammation (Hodge et al, 2005). Interleukin-6 is also known to act as a crucial player in developing chronic inflammation, resulting in immune escape and acceleration of tumour progression (Multhoff and Radons, 2012). The biological effects of IL-6 are well known to be mediated through the activation of signal transducer and activator of transcription 3 (STAT3), and STAT3 has been shown to have a critical role in the interaction between tumour-associated macrophages (TAMs) and tumour cells within the tumour microenvironment, thereby leading to resistance to chemotherapy and radiotherapy (Yu et al, 2007; Masuda et al, 2010; Komohara et al, 2014). Indeed, we previously demonstrated that higher numbers of TAMs contributed to resistance to chemoradiotherapy in OSCC (Matsuoka et al, 2015). We also recently showed that circulating high concentrations of IL-6 were positively correlated with the systemic inflammatory response, such as an elevated neutrophil-tolymphocyte ratio, which predicts a poor response to chemoradiotherapy in OSCC patients (Nakashima et al, 2016). However, the biological roles of IL- 6 on radiosensitivity in OSCC have not yet been fully elucidated.

The Nrf2-Keap1 system is currently recognised as one of the main cellular defence mechanisms against oxidative stress (Taguchi et al, 2011). Under basal conditions, Nrf2 (nuclear factor erythroid 2-related factor 2) is sequestered by the repressor Kelch-like ECHassociated protein1 (Keap1) in the cytoplasm and constitutively degraded through the ubiquitin-proteasome pathway (Taguchi et al, 2011). Under oxidative stress, Nrf2 escapes Keap1-mediated repression and translocates into the nucleus to induce the transcription of numerous cytoprotective genes (Taguchi et al, 2011). The autophagy-adaptor protein p62 interacts with the Nrf2binding site on Keap1 and phosphorylation of p62 markedly increases p62's binding affinity for Keap1, thus inducing the expression of cytoprotective Nrf2 targets (Komatsu et al, 2010; Ichimura et al, 2013). However, recent studies have revealed that the Nrf2-mediated antioxidant pathway is implicated in the radioresistance of cancer cells (Zhou et al, 2013).

Accumulating evidence indicates that IL-6 and the Nrf2mediated antioxidant pathway both contribute to treatment resistance in various cancers (Zhou et al, 2013; Fisher et al, 2014; Komohara et al, 2014; Nakashima et al, 2016). However, the interplay between IL-6 and Nrf2 signalling pathway remains largely unknown. In the present study, we therefore investigated the biological roles of IL- 6 on radiosensitivity and the relationship between IL-6 and Nrf2 activation in OSCC and found that increased levels of IL-6 confer radioresistance by suppressing oxidative stress via the Nrf2-antioxidant pathway in OSCC.

\section{MATERIALS AND METHODS}

Reagents. Recombinant human IL-6 was purchased from Pepro Tech (Rocky Hill, NJ, USA), and the humanised anti-IL-6 receptor
(IL-6R) monoclonal antibody (IgG1 class) tocilizumab was purchased from Chugai Pharmaceutical Co., Ltd (Tokyo, Japan).

Clinical samples from the patients. For the histopathological analysis, primary oral cancer tissue samples were obtained from 22 patients with advanced OSCC treated at Kumamoto University Hospital between October 2003 and October 2009. All of the patients were preoperatively treated with a total dose of $30 \mathrm{~Gy}$ of concurrent chemoradiotherapy followed by curative surgery as a phase II study (Nagata et al, 2011; Yoshida et al, 2014). Radiotherapy was administered at a daily dose of 2 Gy five times a week for 15 days. The study followed the guidelines of the Ethics Committee of Kumamoto University. The nature and aims of the study were explained to all patients, and all gave their informed consent for the research.

Immunohistochemistry. Formalin-fixed specimens were embedded in paraffin, cut into $4 \mu \mathrm{m}$ sections, and mounted on MAS-GP-coated slides (Matunami Glass Ind., LTD, Osaka, Japan). The sections were heated with an autoclave in $10 \mu \mathrm{moll}^{-1}$ citrate buffer ( $\mathrm{pH} 6.0$ or 9.0 ) for $15 \mathrm{~min}$ at $121^{\circ} \mathrm{C}$ for antigen retrieval after deparaffinisation and rehydration. The sections were incubated with $3 \% \mathrm{H}_{2} \mathrm{O}_{2}$ in absolute methanol for 30 min to block endogenous peroxidase activity. Then, nonspecific background staining was blocked using Protein Block Serum Free Reagent (Dako, Glostrup, Denmark) for $15 \mathrm{~min}$, followed by overnight incubation at $4{ }^{\circ} \mathrm{C}$ with anti-human IL-6 (1:50; Abcam, Cambridge, UK), anti-human phospho-STAT3 (Thy705) (1:100; Cell Signaling Technology, Danvers, MA, USA), anti-human IL-6 receptor $\alpha$ (IL-6R $\alpha)(1: 100$; Santa Cruz Biotechnology, Inc., Dallas, TX, USA), anti-human CD163 (1:100; Leica Biosystems, Heidelberger, Germany), anti-human Cytokeratin AE1/AE3 (1:200; Dako), anti-human phospho-Nrf2 (1:100; Abcam), anti-human Mn-SOD (1 : 100; Merck Millipore, Billerica, MA, USA) antibodies diluted in a phosphate-buffered saline (PBS) solution containing $1 \%$ bovine serum albumin (BSA). This was followed by sequential 60 min incubations with the secondary antibodies (EnVision + System-HRP Labelled Polymer; Dako), and visualisation with the Liquid DAB + Substrate Chromogen System (Dako). All of the slides were lightly counterstained with haematoxylin for $30 \mathrm{~s}$ before dehydration and mounting.

Cell lines and cell culture. The human OSCC cell lines derived from the primary tumour, SAS and HSC-2 were obtained from the Japanese Collection of Research Bioresources bank of the National Institutes of Biomedical Innovation, Health and Nutrition (Osaka, Japan), and cultured with DMEM supplemented with $10 \%$ FBS and maintained under humidified $5 \% \mathrm{CO}_{2}$ incubation at $37^{\circ} \mathrm{C}$.

Irradiation. A dose of 6 and $10 \mathrm{~Gy}$ irradiation was performed in a $150-K V p$ X-ray generator (Model MBR-1520R, Hitachi, Tokyo, Japan) with a total filtration of $0.5 \mathrm{~mm}$ aluminium plus $0.1 \mathrm{~mm}$ copper filter. The dose rate measured by a thimble ionisation chamber (IC 17A, Far West Technology, Goleta, CA, USA) was $1.01 \mathrm{~Gy} \mathrm{~min}^{-1}$.

Cell proliferation assay. To assess the normal proliferation, viable cells under IL- 6 treatment $\left(0,50\right.$, and $\left.200 \mathrm{pg} \mathrm{ml}^{-1}\right)$ with or without irradiation $(0,2,6$, and $10 \mathrm{~Gy})$ were quantified every $24 \mathrm{~h}$ using the Cell Counting Kit-8 (Dojindo, Kumamoto, Japan).

Enzyme-linked immunosorbent assay for the detection of secreted IL-6. The cells $\left(2 \times 10^{5}\right)$ were seeded in a $60 \mathrm{~mm}$ tissue culture dish (Asahi Techno Glass Co., LTD, Shizuoka, Japan) and incubated in DMEM with $1 \%$ FBS for $24 \mathrm{~h}$. The cells were then irradiated with 6 and 10 Gy of X-rays. At 12, 24, 36, 48, and $60 \mathrm{~h}$ after irradiation, the conditional culture media were collected, and the concentration of IL-6 was measured using a Human IL-6 Quantikine enzyme-linked immunosorbent assay (ELISA) Kit 
(R\&D Systems, Minneapolis, MN, USA) in accordance with the manufacturer's instructions.

RNA isolation and real-time quantitative PCR. Total RNA was isolated from the treated cells using mirVana miRNA Isolation Kit (Life Technologies, Palo Alto, CA, USA). Total RNA was then reverse-transcribed to cDNA using a ReverTra qPCR RT Kit (Toyobo, Osaka, Japan). Each PCR reaction was run using a Thunderbird SYBR qPCR Mix (Toyobo) on a Light Cycler 1.5 (Roche, Indianapolis, IN, USA). The comparative Ct $(\Delta \Delta \mathrm{Ct})$ method was used to determine the fold changes in the expression using glyceraldehyde-3-phospate dehydrogenase (GAPDH). Each sample was run in triplicate. The following primers were used: Keap1 (forward, 5'-GGGTCCCCTACAGCCAAG-3'; reverse, 5'-T GGGGTTCCAGAAGATAAGC-3'); GAPDH (forward, 5'-CAACAGCCTCAAGATCATCAGC-3'; reverse, $5^{\prime}$-TTCTAGACGGCAGGTCAGGTC-3'). The cycling conditions were as follows: initial denaturation at $98^{\circ} \mathrm{C}$ for $5 \mathrm{~min}$ followed by 45 cycles at $98^{\circ} \mathrm{C}$ for $15 \mathrm{~s}, 58^{\circ} \mathrm{C}$ for $30 \mathrm{~s}$, and $72^{\circ} \mathrm{C}$ for $60 \mathrm{~s}$. These experiments were performed in triplicate.

The modified high-density survival assay. The modified highdensity survival (HDS) assay was performed according to the method of Kuwahara et al (2010). Exponentially growing cells $\left(5 \times 10^{5}\right)$ were seeded in a $60 \mathrm{~mm}$ tissue culture dish (Asahi Techno Glass Co., LTD) and incubated in DMEM with 1\% FBS for $48 \mathrm{~h}$. The cells treated with various concentrations of IL-6 (0, 50, and $200 \mathrm{pg} \mathrm{ml}^{-1}$ ) were then exposed to $6 \mathrm{~Gy}$ of X-rays. After $72 \mathrm{~h}$ incubation, $10 \%$ of the cells in each flask were seeded in a new $60 \mathrm{~mm}$ culture dish with various concentrations of IL-6 and incubated for a further $72 \mathrm{~h}$. Finally, the total number of cells in each culture dish was counted via the Trypan blue dye exclusion test, and the survival of the cells was plotted. Similarly, $20 \mathrm{ng} \mathrm{ml}^{-1}$ tocilizumab was added to the cells in the modified HDS assay.

Clonogenic assay. After a single exposure to $6 \mathrm{~Gy}$ of X-rays, the cells $\left(1 \times 10^{3}\right)$ were seeded in a $60 \mathrm{~mm}$ culture dish coated with gelatin (Asahi Techno Glass Co., LTD) under treatment with control agents or $100 \mathrm{pg} \mathrm{ml}^{-1}$ IL-6 and incubated in DMEM with $1 \%$ FBS for 10 days. After 10 days, the cells were fixed with $99.5 \%$ methanol and stained with Giemsa solution (Wako, Osaka, Japan).

Immunofluorescent staining and evaluation. The cells $\left(2 \times 10^{4}\right)$ were seeded onto glass slides (Merck Millipore) and incubated in DMEM with $1 \%$ FBS for $24 \mathrm{~h}$. Then, IL-6 at $200 \mathrm{pg} \mathrm{ml}^{-1}$ and tocilizumab at $20 \mathrm{ng} \mathrm{ml}^{-1}$ were added to the cells, and the cells were exposed to $10 \mathrm{~Gy}$ of X-rays. After 24 ( $\gamma$-H2AX) or $48 \mathrm{~h}$ (Nrf2, Keap1, and phospho-p62) incubation, the cells were fixed in $4 \%$ paraformaldehyde in a PBS solution for $30 \mathrm{~min}$. The cells were then washed with PBS-T for $5 \mathrm{~min}$ and blocked with PBS-T with $5 \%$ BSA for 5 min on ice. After blocking, the cells were incubated for $2 \mathrm{~h}$ at room temperature, with the primary antibodies against phosphorylated histone $\mathrm{H} 2 \mathrm{AX}(\gamma-\mathrm{H} 2 \mathrm{AX})(1: 00$; Merck Millipore), Nrf2 (1:50; Abcam), Keap1 (1:400; Cell Signaling Technology), and phospho-p62 (1:5000; Medical \& Biological Laboratories Co., LTD, Woburn, MA, USA) in PBS-T with $1 \%$ BSA. Subsequently, the glass slides were washed with PBS-T three times ( 5 min each), and the cells were incubated for $1.5 \mathrm{~h}$ at room temperature with the secondary antibodies (Alexa Fluor 488 and 594 donkey anti-rabbit and/or anti-mouse IgG; Life Technologies) in PBS-T with 1\% BSA. After being washed with PBS, the cells were counterstained and mounted with Vectashield (Vector Laboratories, Inc., Burlingame, CA, USA) and examined with a fluorescent microscope (Model BZ-X700; KEYENCE, Osaka, Japan). About evaluating the $\gamma$-H2AX foci, if the cell had $\geqslant 10 \gamma$-H2AX foci per nucleus, the cell was considered to be positive for $\gamma-\mathrm{H} 2 \mathrm{AX}$ (Taneja et al, 2004).
Western blot analysis. The whole-cell and nuclear protein (Minute Cytoplasmic and Nuclear Extraction Kits; Invent Biotechnologies, Inc., Eden Prairie, MN, USA) were separated using 7.5 or $12.5 \%$ SDS-PAGE, transferred onto nitrocellulose membranes, and probed with antibodies against phospho-Nrf2 (S40; 1:15000; Abcam), phospho-STAT3 (Thy705; 1:500; Cell Signaling Technology), Keap1 (1:1000; Cell Signaling Technology), phospho-p62 (1:1000; Medical \& Biological Laboratories Co., LTD), Lamin B1 (1:10 000; Abcam), and $\beta$-actin ( $1: 10000$; Sigma, St Louis, MO, USA). After overnight incubation at $4{ }^{\circ} \mathrm{C}$, the membranes were washed and incubated with appropriate horseradish peroxidase-conjugated secondary antibodies and visualised using the ECL prime detection kit (GE Healthcare, Buckinghamshire, UK).

Immunoprecipitation. The cells proteins were extracted in RIPA buffer (25 mM Tris $\cdot \mathrm{HCl}$ (pH 7.6), $150 \mathrm{~mm} \mathrm{NaCl}, 1 \% \mathrm{NP}-40,1 \%$ sodium deoxycholate, and $0.1 \%$ SDS) containing a protease inhibitor cocktail (Roche Diagnostics). Then, the total proteins were cleaned and incubated with $5 \mu \mathrm{g}$ of specific antibody against Keap1 to form immunocomplexes, which were precipitated with protein A-Sepharose (GE Healthcare). Finally, the presence of immunocomplexes was detected by a western blotting analysis.

Cellular ROS/superoxide detection assay. The cells $\left(2 \times 10^{4}\right)$ were seeded onto 24-well plates (Asahi Techno Glass Co., LTD) and incubated in DMEM with 1\% FBS for $24 \mathrm{~h}$. Next, IL- 6 at 0 and $200 \mathrm{pg} \mathrm{ml}^{-1}$ and tocilizumab at $20 \mathrm{ng} \mathrm{ml}^{-1}$ were added, and the cells were exposed to $10 \mathrm{~Gy}$ of X-rays. After $48 \mathrm{~h}$ incubation, the appearance of ROS/superoxide in the cells was detected using Cellular ROS/Superoxide Detection Assay Kit (Abcam). The observations to obtain these findings were done under a fluorescent microscope.

Statistical analysis. The differences in the mean values between the two groups were statistically analysed using Student's $t$-test, whereas the differences in the mean values among multiple groups were analysed using a one-way ANOVA with the Bonferroni/Dunn test. All $P$-values were based on two-tailed statistical analyses, and $P$-values $<0.05$ were considered to be statistically significant $\left({ }^{\star} P<0.05\right.$ and $\left.{ }^{\star *} P<0.01\right)$.

\section{RESULTS}

IL-6 levels in the tumour microenvironment of irradiated OSCC tissues. To examine the clinical significance of IL- 6 in the tumour microenvironment, we assessed the expression levels of IL- 6 by immunohistochemistry using surgical tissue specimens, which contained surviving OSCC cells after preoperative $30 \mathrm{~Gy}$ of chemoradiotherapy. Representative photographs illustrating the expression patterns of various molecules are shown in Figure 1. As shown in Figure 1B, increased levels of IL-6 were observed in the tumour cells and stromal cells. Among the stromal cells, CD163positive M2 macrophages and TAMs around tumour cells overexpressed IL-6 (Figure 1B and F). In addition, high expression levels of IL-6 signalling molecules, such as IL-6R $\alpha$ and phosphoSTAT3, were also observed in AE1/AE3-positive tumour cells (Figure 1E) and the surrounding stromal cells (Figure 1C and D). These results suggest that increased levels of IL-6 and IL-6 signalling may promote the development of radioresistance in both autocrine and paracrine manners in the tumour microenvironment of OSCC tissues.

IL-6 effect on the radiation-induced cell death of OSCC cells. To clarify the biological impacts of IL-6 in the tumour microenvironment, we performed in vitro experiments. First, we examined the effects of IL-6 in irradiated OSCC cells using a modified HDS assay. Irradiated OSCC cells under IL- 6 treatment showed significantly lower radiosensitivity than the control cells (Figure 2A and B). 

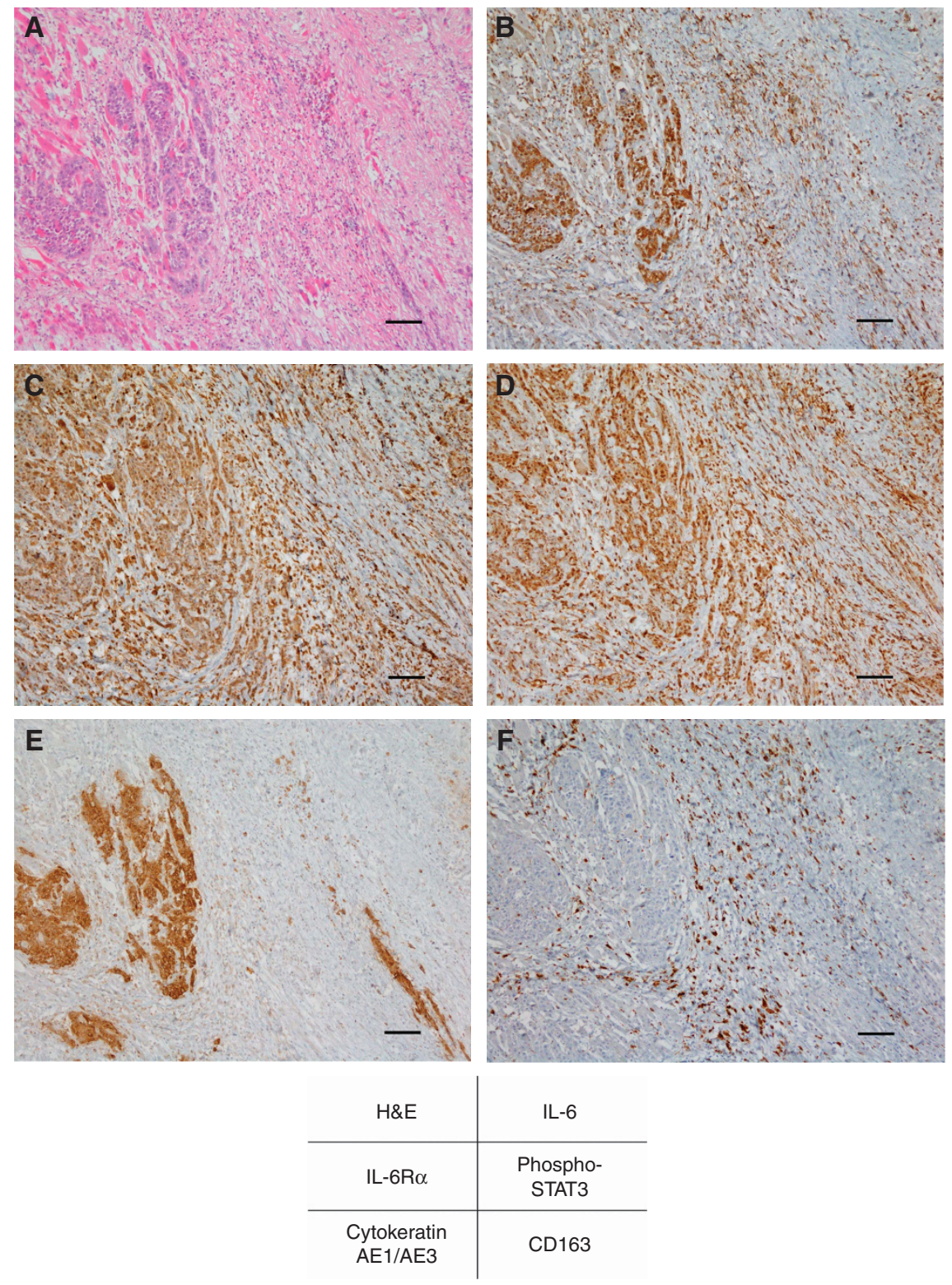

Figure 1. Interleukin-6 levels are increased in the tumour microenvironment of irradiated OSCC tissues. Representative microscopic images of H\&E (A) and immunohistochemical staining of IL-6 (B), IL-6R $($ (C), phospho-STAT3 (D), AE1/AE3 (E), and CD163 (F). AE1/AE3 and CD163 were used as surrogate markers for tumour cells and TAMs, respectively. Scale bar, $100 \mu \mathrm{m}$.

Regarding the radioprotective effect of IL-6, the same result was confirmed by a clonogenic assay (Figure $2 \mathrm{C}$ and $\mathrm{D}$ ). We then examined the cellular growth activities of the OSCC cells with or without IL-6 treatment by a cell proliferation assay. Interleukin-6 had no significant effect on the cell proliferation in any OSCC cells, regardless of irradiation (Supplementary Figure 1), indicating that the radioresistance elicited by IL- 6 is not due to an increased cell proliferation. We also examined the amount of extracellularly released IL- 6 in the conditioned media at $48 \mathrm{~h}$ after X-ray irradiation without IL-6 treatment using an ELISA kit (Supplementary Figure 2A and B). Despite the lack of marked differences in cell proliferation between X-ray-irradiated and non-irradiated cells at $48 \mathrm{~h}$ after irradiation, the release of IL- 6 in the irradiated OSCC cells was significantly higher than that observed in the non-irradiated cells. Furthermore, a significant level of IL-6R $\alpha$ expression was confirmed in these cell lines at both the gene and protein levels (data not shown). These results suggest that extracellularly released IL-6 from OSCC cells after irradiation may contribute to radioresistance in an autocrine manner.
Effect of IL-6 signalling blockade on OSCC cell radiosensitisation. Next, we confirmed whether or not the blockade of IL-6 signalling affects radiosensitivity in irradiated OSCC cells. We examined the radiosensitivity of irradiated OSCC cells under treatment with either or both IL-6 or the humanised anti-human IL-6R antibody tocilizumab using a modified HDS assay. The photographs under phase-contrast microscopy show the surviving cells after radiation under each treatment condition (Figure $3 \mathrm{~A}$ and $\mathrm{C}$ ). The addition of tocilizumab to IL- 6 or to the control agent significantly decreased the surviving fraction compared with IL-6 or control alone (Figure $3 \mathrm{~B}$ and $\mathrm{D}$ ). These results suggest that the blockade of IL-6 signalling sensitises OSCC cells to radiation.

Contribution of IL-6 signalling to decreased DNA damage in the irradiated OSCC cells. Next, we investigated whether or not IL-6 affects the number of double-stranded DNA breaks in irradiated OSCC cells. $\gamma$-H2AX was used to quantitate the double-stranded DNA breaks attributed to X-ray irradiation. We identified the cells with $\geqslant 10 \gamma-\mathrm{H} 2 \mathrm{AX}$ foci by counting at $1,3,6,24$, and $48 \mathrm{~h}$ after 



Figure 2. Interleukin-6 suppresses the radiation-induced cell death of OSCC cells. The survival fraction of SAS cells (A) and HSC-2 cells (B) after exposure to 6 Gy of X-rays was evaluated by a modified HDS assay under various concentrations of IL-6 $\left(0,50\right.$, and 200 pg ml $\left.{ }^{-1}\right)$. The results are shown as the means \pm s.d. of three independent experiments. ${ }^{\star \star} P<0.01$. ( $\mathbf{C}$ and $\mathbf{D}$ ) The photographs of the cell survival fraction as evaluated by a clonogenic assay. After exposure to 6 Gy of X-rays, the SAS and HSC-2 cells were seeded in 60 mm culture dishes under treatment with 0 or $100 \mathrm{pg} \mathrm{ml}^{-1} \mathrm{IL-6}$, and incubated. After 10 days' culture, the cells were fixed and visualised.

10 Gy irradiation (Taneja et al, 2004; Kuwahara et al, 2009). The frequency of $\gamma$-H2AX-positive SAS cells under IL-6 (20.0\% $\pm 3.1 \%)$ was lower than that under control conditions $(68.5 \% \pm 7.3 \%)$ at $24 \mathrm{~h}$ after irradiation (Figure $4 \mathrm{~A}$ and B). However, the frequency of $\gamma$-H2AX-positive cells under tocilizumab alone or IL-6 plus tocilizumab treatment was higher than that under control conditions (Figure 4C) or IL-6 treatment alone (Figure 4D). In addition, IL- 6 treatment significantly reduced the number of double-stranded DNA breaks from the early stage to $48 \mathrm{~h}$ after irradiation (Figure 4E).

Effect of IL-6 on the activation of the Keap1/Nrf2 system in the irradiated OSCC cells. As recent studies have revealed that Nrf2 is involved in cancer radioresistance, we next examined the effect of IL- 6 on the activation of Nrf2 in OSCC cells. As shown in Figure 5A, a subtle increase in the Nrf2 expression in the cytoplasm and nucleus was observed in the cells after $10 \mathrm{~Gy}$ irradiation by immunofluorescence. In addition, IL-6 treatment significantly increased the expression level of Nrf2 in the cytoplasm and nucleus of the irradiated cells (Figure 5A). Western blot analyses showed that IL- 6 activated not only the downstream molecule STAT3 but also Nrf2 in the irradiated cells (Figure 5B). In addition, IL-6 treatment of irradiated OSCC cells did not change the mRNA or protein levels of Keap1, an inhibitor of Nrf2, but increased the protein level of phospho-p62 (Figure 5C and D). These data suggest that IL- 6 promotes Nrf2 activation by upregulating the interaction between Keap1 and p62 in the irradiated cells. Associated with this phenomenon, immunofluorescence of the irradiated cells revealed a higher number of colocalisations of Keap1 and p62 under IL-6 treatment (Figure 5E). Reflecting these results, the dissociation between Keap1 and Nrf2 was observed in the IL-6-treated irradiated cells (Figure 5F).

Effect of IL-6 on the oxidative stress regulation in the irradiated OSCC cells. Next, we investigated whether or not IL-6 modulates oxidative stress via Nrf2-antioxidant pathway in the irradiated OSCC cells. We examined the expression of Mn-SOD, an Nrf2dependent ROS scavenger ( $\mathrm{Na}$ et al, 2008), in irradiated cells under IL-6 treatment. Western blot analyses revealed that IL- 6 enhances the expression of Mn-SOD via Nrf2 activation, while tocilizmab inhibits the expression (Figure 6A). Reflecting these results, IL-6 decreased the amount of ROS/superoxide, and tocilizumab 
A

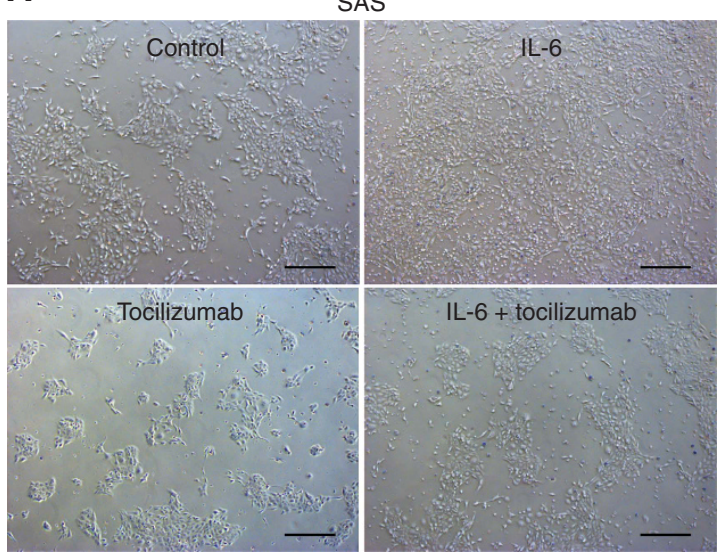

C

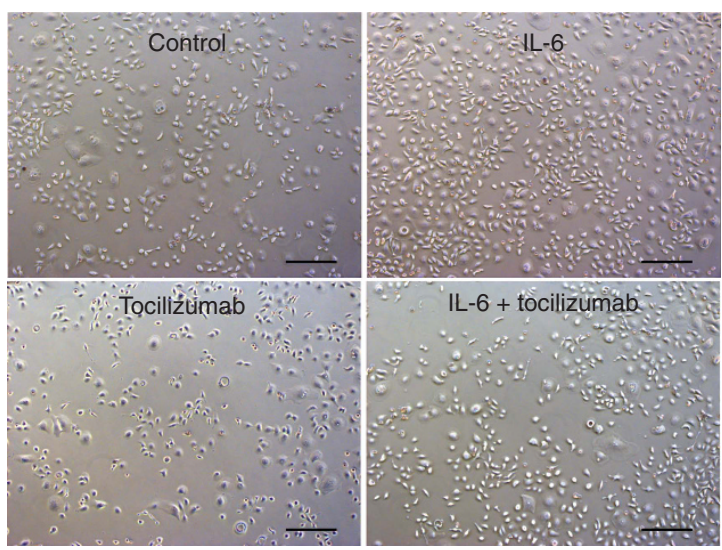

B

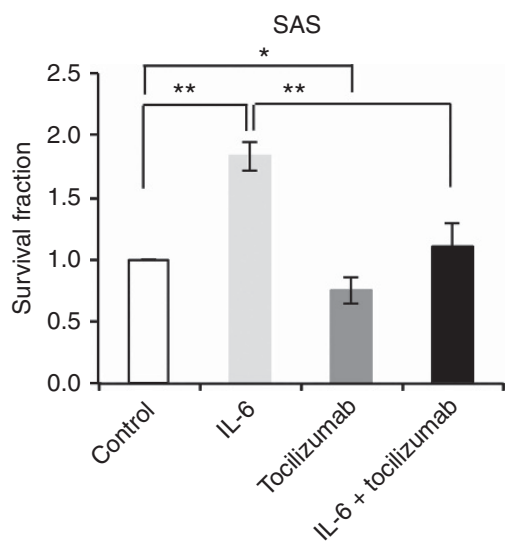

D

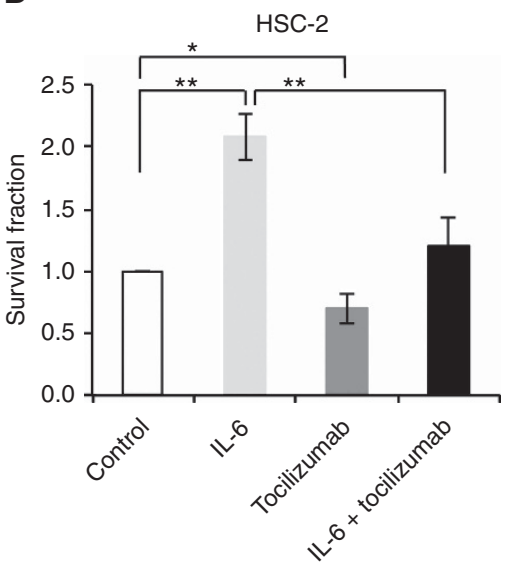

Figure 3. Blockade of IL-6 signalling sensitises OSCC cells to radiation. (A and $\mathbf{B}$ ) The modified HDS assay using SAS cells under control conditions, or $200 \mathrm{pg} \mathrm{ml}^{-1} \mathrm{IL-6}$, or $20 \mathrm{ng} \mathrm{ml}^{-1}$ tocilizumab, or $200 \mathrm{pg} \mathrm{ml}^{-1} \mathrm{IL}-6$ plus $20 \mathrm{ng} \mathrm{ml}^{-1}$ tocilizumab. The cells were exposed to $6 \mathrm{~Gy}$ of X-rays. The images of the survival fraction of SAS cells under phase-contrast microscopy (A) and the corresponding frequency graph (B). (C and $\mathbf{D})$ The modified HDS assay using HSC-2 cells under control conditions, or $200 \mathrm{pg} \mathrm{ml}^{-1} \mathrm{LL}-6$, or $20 \mathrm{ng} \mathrm{ml}^{-1}$ tocilizumab, or $200 \mathrm{pg} \mathrm{ml}^{-1} \mathrm{IL}-6 \mathrm{plus}$ $20 \mathrm{ng} \mathrm{ml}^{-1}$ tocilizumab. The cells were exposed to $6 \mathrm{~Gy}$ of X-rays. The images of the survival fraction of HSC-2 cells under phase-contrast microscopy $(\mathbf{C})$ and the corresponding frequency graph (D). The results are shown as the means \pm s.d. of three independent experiments. ${ }^{\star} P<0.05$ and ${ }^{* *} P<0.01$. Scale bar, $200 \mu \mathrm{m}$.

inhibited these effects (Figure 6B). We also confirmed the involvement of the IL-6/Nrf2-antioxidant pathway in some specimens from patients with OSCC that showed resistant to $30 \mathrm{~Gy}$ of chemoradiotherapy. The representative results of immunohistochemical staining for IL-6, phospho-Nrf2, and MnSOD are shown in Figure 6C. As expected, the expression levels of IL-6, phospho-Nrf2, and Mn-SOD were increased in almost all specimens. We summarised the experimental evidence of IL-6mediated radioresistance model in the tumour microenvironment of irradiated OSCC tissue based on our data in Figure 6D.

\section{DISCUSSION}

In the present study, we observed a number of meaningful findings. First, an increased level of IL- 6 conferred radioresistance to OSCC cells by protecting them from oxidative damage. Second, IL-6 activated the Nrf2-antioxidant pathway. To our knowledge, no other reports have identified the molecular mechanisms underlying IL-6-mediated radioresistance in OSCC. In addition, given the growing evidence that upregulated IL- 6 in the tumour microenvironment promotes malignant phenotypes, leading to treatment resistance (Guo et al, 2012; Fisher et al, 2014; Komohara et al, 2014), it is noteworthy that we uncovered the interplay between IL-6 and the Nrf2-antioxidant pathway, which will help develop novel IL-6-targeting therapies.

There have been some reports showing an association between IL-6 and the radioresistance of cancer cells (Dubost et al, 2002; Chen et al, 2010, 2012, 2015; Bochet et al, 2011; Wu et al, 2013). Two reports on prostate cancer (Wu et al, 2013) and liver cancer (Chen et al, 2012) cited findings consistent with our own, showing that IL-6 protects cancer cells from radiation-induced oxidative DNA damage. In addition, one report in lung cancer further showed that IL-6 signalling promotes DNA repair in CD133positive cancer stem cell-like cells (Chen et al, 2015). As we did not evaluate the effects of IL-6 on DNA repair, whether or not IL-6 promotes DNA repair in OSCC remains unknown. However, our present findings suggest that IL- 6 could be a potential target for overcoming radioresistance in many cancers.

Radiation is known to significantly contribute to the generation of ROS in various cells, and it can induce DNA damage and oxidative stress responses (Lawenda et al, 2008). In contrast, Nrf2 is a key transcriptional regulator of genes encoding numerous cytoprotective enzymes in response to oxidative stress, such as excessive ROS (Zhou et al, 2013). Among such Nrf2-dependent cytoprotective enzymes, $\mathrm{Mn}$-SOD is crucial for radioresistance, due to its ability to scavenge ROS and superoxide (Fisher and Goswami, 

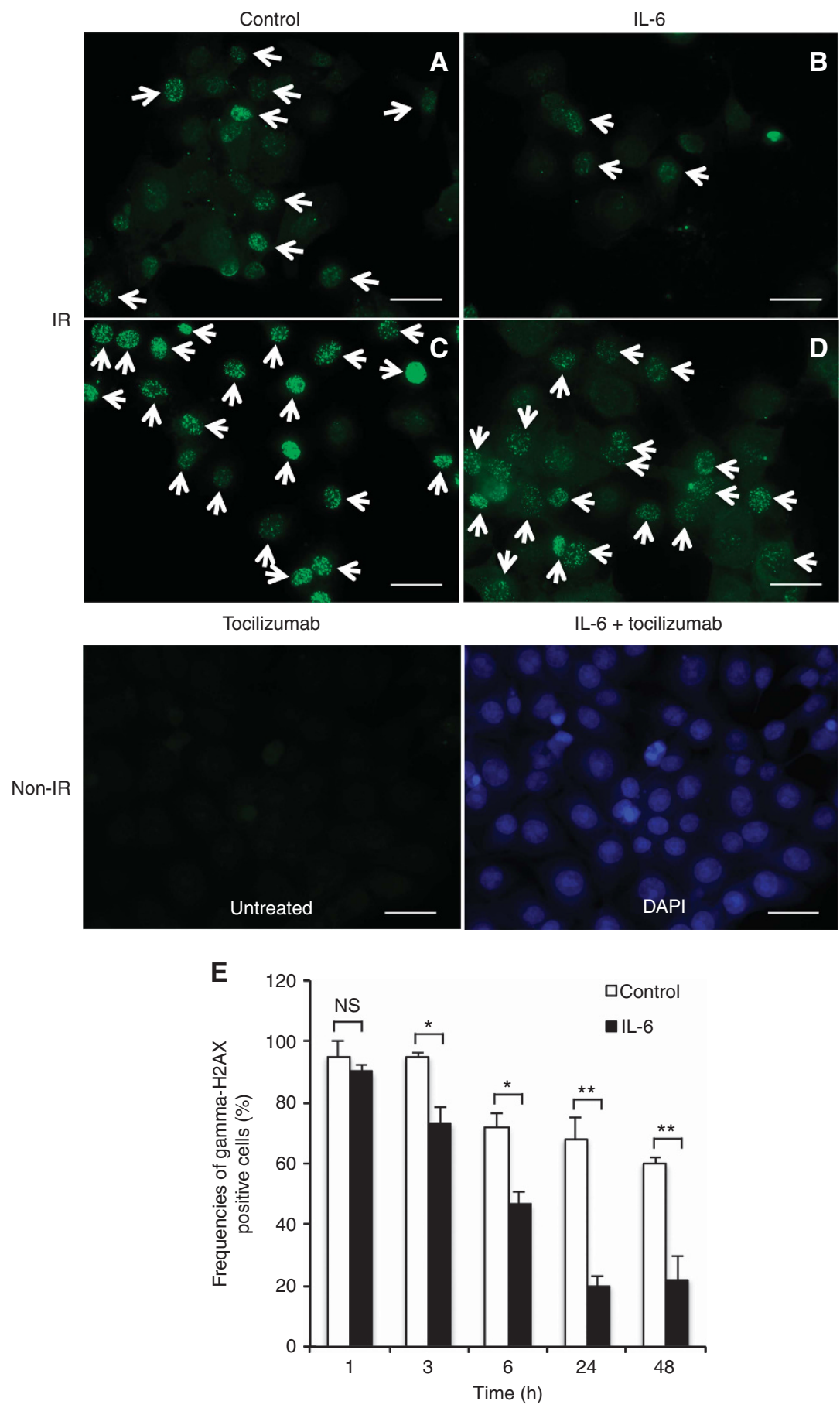

Figure 4. Interleukin-6 signalling helps reduce DNA damage in irradiated OSCC cells. (A-D) Representative images of immunofluorescence of $\gamma$ - $\mathrm{H} 2 \mathrm{AX}$ foci under various conditions after exposure to $10 \mathrm{~Gy}$ of $\mathrm{X}$-rays. The images of $\gamma$-H2AX foci in SAS cells are shown at $24 \mathrm{~h}$ under treatment with control (A), or $200 \mathrm{pg} \mathrm{ml}^{-1} \mathrm{IL}-6$ (B), or $20 \mathrm{ng} \mathrm{ml}^{-1}$ tocilizumab (C), or $200 \mathrm{pg} \mathrm{ml}^{-1} \mathrm{IL-6}$ plus $20 \mathrm{ng} \mathrm{ml}^{-1}$ tocilizumab (D) after irradiation. The arrows indicate the cells with $\geqslant 10 \gamma$-H2AX foci. (E) Frequencies of $\gamma-\mathrm{H} 2 \mathrm{AX}$-positive cells at each time after exposure to $10 \mathrm{~Gy}$ of $\mathrm{X}$-rays under treatment with control or $200 \mathrm{pg} \mathrm{ml}^{-1} \mathrm{IL}-6$. The results are shown as the means \pm s.d. of three independent experiments. ${ }^{\star} P<0.05$ and ${ }^{\star \star} P<0.01$. Scale bar, $50 \mu \mathrm{m}$.

2008; Zhang and Martin, 2014). Indeed, as shown in Figure 6A and B in our study, Nrf2-dependent Mn-SOD expression levels have been linked to the ability to scavenge ROS and superoxide. These results suggest that radiosensitivity in OSCC cells can be regulated by the Nrf2-antioxidant pathway, a notion supported by evidence showing that the suppression of the Nrf2-antioxidant pathway sensitises tumour cells to radiation in lung cancer (Lee et al, 2012; Liu et al, 2016; Zhao et al, 2016), breast cancer (McDonald et al, 2010), and prostate cancer (Zhang et al, 2010).
Radiation therapy promotes inflammatory responses in the tumour tissues, and the upregulation of cytokines such as IL-6 is not only a crucial mediator of inflammation but also confers a survival advantage to tumour cells (Deorukhkar and Krishnan, 2010). Regarding the interplay between IL-6 and the Nrf2 signalling pathway, to our knowledge, no one has demonstrated that IL-6 activates the Nrf2-antioxidant pathway after radiation against OSCC cells. To test our hypothesis, in addition to evaluating the Nrf2 activation via western blot (Figure $5 \mathrm{~B}$ and $\mathrm{D}$ ) and 

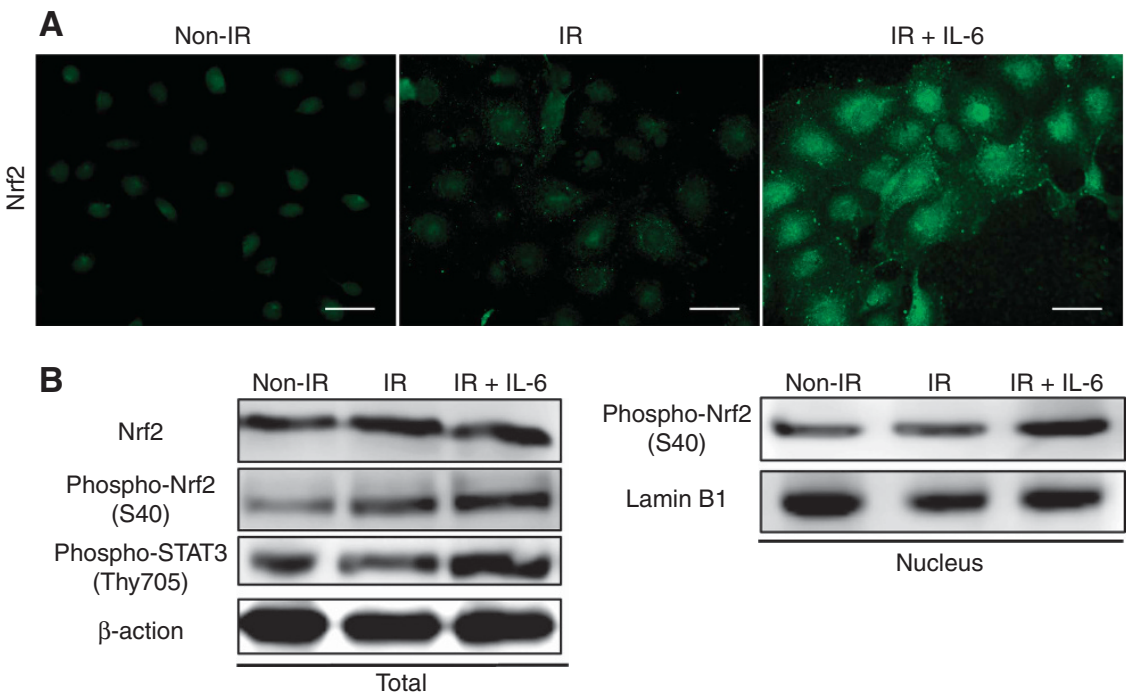

C

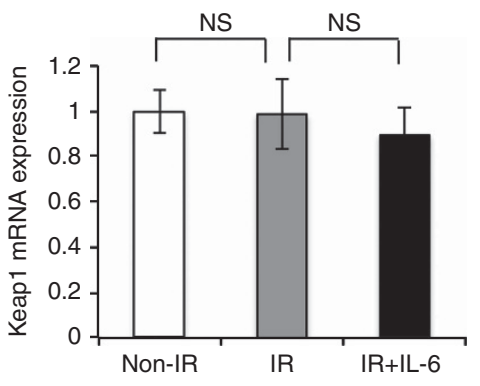

D



E IR $\mathrm{IR}+\mathrm{IL}-6$
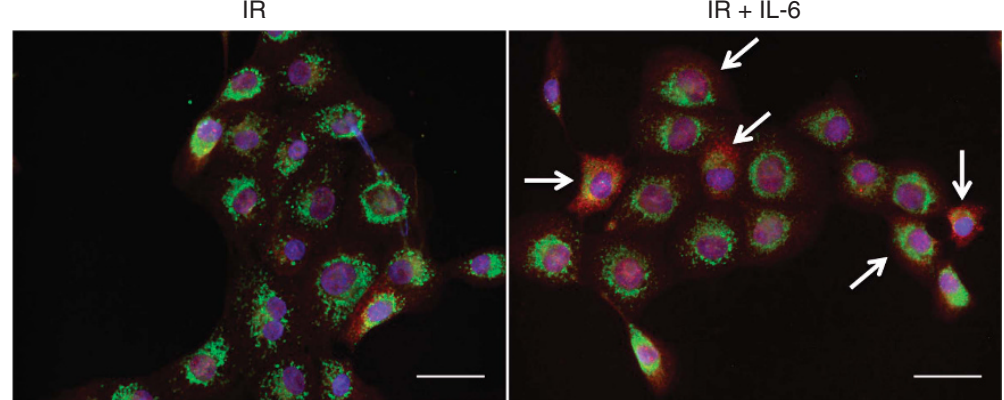

Green, Keap1; Red, Phospho-p62; Blue, DAPI



Figure 5. Interleukin-6 activates the Keap1/Nrf2 system in irradiated OSCC cells. (A) The images of immunofluorescence of Nrf2 in SAS cells at $48 \mathrm{~h}$ after non-X-ray or $10 \mathrm{~Gy}$ of X-ray irradiation with or without $200 \mathrm{pg} \mathrm{ml}^{-1} \mathrm{IL}-6$. (B) The protein levels of Nrf2, phospho-Nrf2, and phosphoSTAT3 in SAS cells at $48 \mathrm{~h}$ after non-X-ray or $10 \mathrm{~Gy}$ of X-ray irradiation with or without $200 \mathrm{pg} \mathrm{ml}^{-1} \mathrm{IL}-6$. The whole-cell and nuclear protein were prepared, and the expression of Nrf2, phospho-Nrf2, and phospho-STAT3 proteins was examined using a western blot analysis. (C) The expression of Keap1 mRNA in SAS cells at $48 \mathrm{~h}$ after non-X-ray or $10 \mathrm{~Gy}$ of X-ray irradiation with or without $200 \mathrm{pg} \mathrm{ml}^{-1} \mathrm{IL}-6$. (D) The protein levels of Keap1 and phospho-p62 in SAS cells. The western blot analysis was done using the same lysate as B. (E) The images of immunofluoresent double staining of Keap1 and phospho-p62 in SAS cells at $48 \mathrm{~h}$ after $10 \mathrm{~Gy}$ of X-ray irradiation with or without $200 \mathrm{pg} \mathrm{ml}^{-1} \mathrm{IL}-6$. The arrows indicate the activated phospho-p62. (F) The Nrf2/Keap1 complex formation detected by an immunoprecipitation analysis in SAS cells. After the same treatment as B, the cell lysates were immunoprecipitated using anti-Keap1 antibody. The immunoprecipitates were immunoblotted with anti-Nrf2 antibody.

immunoprecipitation (Figure 5F) analyses, we confirmed the subcellular localisation of $\mathrm{Nrf} 2$ and $\mathrm{Nrf} 2$ activation-related molecules (Keap1 and p62) in the cells by immunofluorescence (Figure 5A and E). However, IL-6 is known to activate STAT3, which has a crucial role in radiation-induced inflammatory responses (Multhoff and Radons, 2012). Therefore, the effects of radioresistance observed with increased levels of IL- 6 may be brought about by not only Nrf2- but also STAT3-regulated function. However, we believe the novel finding that an IL-6mediated Nrf2-antioxidant pathway is involved in radioresistance 
A

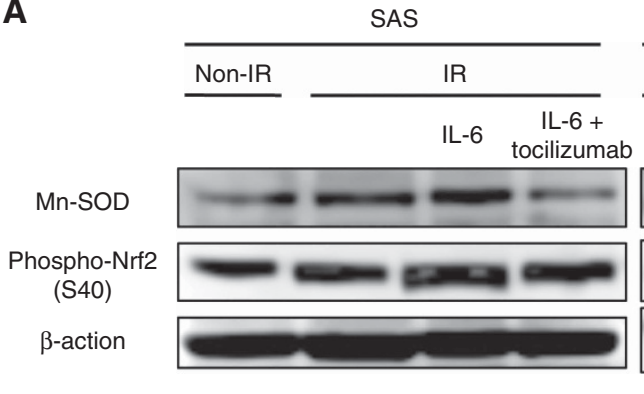

B

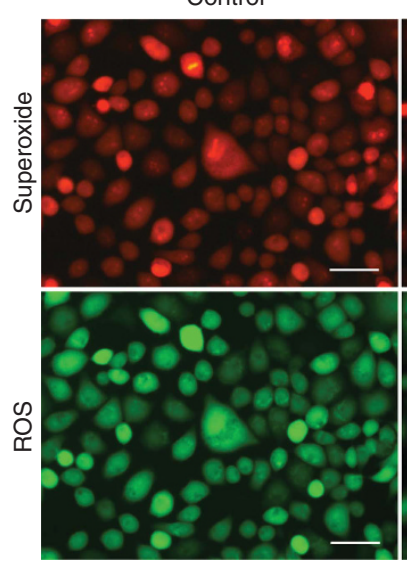

IL-6

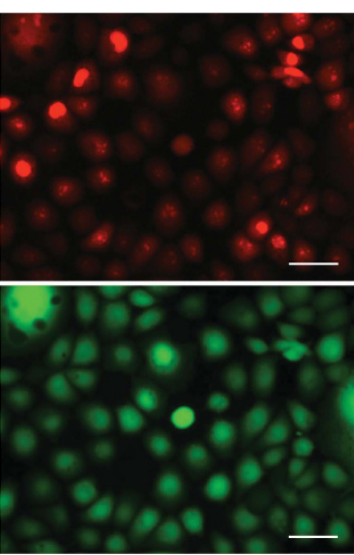

HSC-2
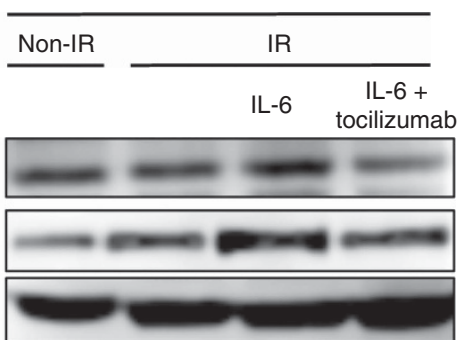

\section{IL-6 + tocilizumab}

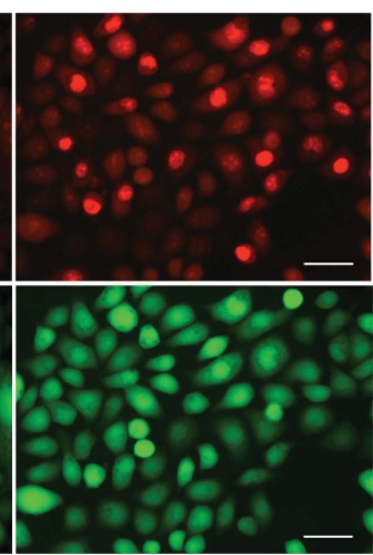

X-ray irradiation

\section{C}

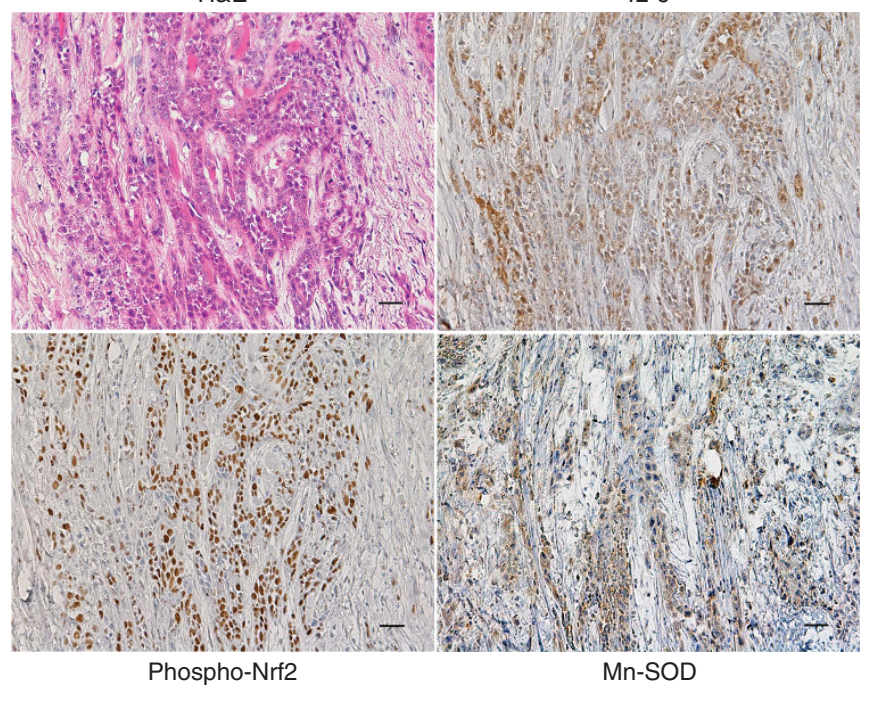

D

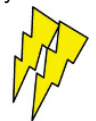

Tumour microenvironment



Figure 6. Interleukin-6 reduces oxidative stress via the Nrf2-antioxidant pathway in irradiated OSCC cells. (A) At $48 \mathrm{~h}$ after non-irradiation or $10 \mathrm{~Gy}$ of X-ray irradiation with vehicle or $200 \mathrm{pg} \mathrm{ml}^{-1} \mathrm{IL}-6$, or $200 \mathrm{pg} \mathrm{ml}^{-1} \mathrm{IL}-6$ plus $20 \mathrm{ng} \mathrm{ml}^{-1}$ tocilizumab, whole-cell lysates in OSCC cells were prepared, and the expression of Mn-SOD and phospho-Nrf2 was examined by a western blot analysis. (B) At $48 \mathrm{~h}$ after $10 \mathrm{~Gy}$ of X-ray irradiation with vehicle or $200 \mathrm{pg} \mathrm{ml}^{-1} \mathrm{IL}-6$, or $200 \mathrm{pg} \mathrm{ml}^{-1} \mathrm{IL}-6$ plus $20 \mathrm{ng} \mathrm{ml}^{-1}$ tocilizumab, the superoxide/ROS were examined in SAS cells using a Cellular ROS/Superoxide Detection Assay Kit. Scale bar, $50 \mu \mathrm{m}$. (C) Representative microscopic images of H\&E and immunohistochemical staining of IL-6, phospho-Nrf2, and Mn-SOD. Scale bar, $25 \mu \mathrm{m}$. (D) A schematic illustration of the IL-6-mediated radioresistance model. When OSCC tissue is exposed to X-ray irradiation, the increased level of IL-6 promotes the dissociation of Keap1 and Nrf2 via binding of Keap1 to phospho-p62, leading to Nrf2 activation. At the same time, the IL-6/STAT3 pathway is also activated. The activated Nrf2 and STAT3 then translocate to the nucleus and induce the expression of antioxidant enzymes such as Mn-SOD, and IL-6. The overexpressed IL-6 helps increase the resistance to radiation in both autocrine and paracrine manners in the tumour microenvironment. 
will prove of great import when targeting inflammatory networks to counter tumour radiosensitisation.

These present findings suggest that targeting the IL- 6 signalling pathway combined with conventional radiotherapy could be a promising new approach for overcoming radioresistance in patients with OSCC. However, an inherent limitation of our study is that the novel findings obtained were based on mainly in vitro data, except for the immunohistochemical analysis using OSCC tissues. Therefore, further studies are needed to confirm the effects of combination therapy with targeting of IL- 6 signalling and radiation using in vivo models. Regarding this point, given our previous finding that the therapeutic approach targeting IL-6R by tocilizumab is effective for OSCC treatment using an in vivo mouse model, we believe that tocilizumab might be useful for preclinically verifying our idea for tumour radiosensitisation (Shinriki et al, 2009, 2011). Furthermore, tocilizumab has been approved for rheumatoid arthritis (RA) in more than 90 countries worldwide and is known to be very effective in the treatment of RA. Therefore, if preclinical findings using tocilizumab are promising in the cancer field, tocilizumab would be useful for clinical trial design from the viewpoint of drug repositioning.

In conclusion, we demonstrated for the first time that IL-6 controls resistance to radiation by suppressing oxidative stress via the Nrf2-antioxidant pathway in cancer. Our data indicate that blockade of IL-6 signalling in combination with conventional radiotherapy could augment the treatment response in patients with radioresistant OSCC, thereby improving the survival rate.

\section{ACKNOWLEDGEMENTS}

This work was supported by JSPS KAKENHI Grant Number 15K11296. We thank Professor Brian Quinn for proofreading the manuscript.

\section{CONFLICT OF INTEREST}

The authors declare no conflict of interest.

\section{REFERENCES}

Bochet L, Meulle A, Imbert S, Salles B, Valet P, Muller C (2011) Cancerassociated adipocytes promotes breast tumour radioresistance. Biochem Biophys Res Commun 411(1): 102-106.

Chen CC, Chen WC, Lu CH, Wang WH, Lin PY, Lee KD, Chen MF (2010) Significance of interleukin-6 signaling in the resistance of pharyngeal cancer to irradiation and the epidermal growth factor receptor inhibitor. Int J Radiat Oncol Biol Phys 76(4): 1214-1224.

Chen MF, Hsieh CC, Chen WC, Lai CH (2012) Role of interleukin-6 in the radiation response of liver tumours. Int J Radiat Oncol Biol Phys 84(5): e621-e630.

Chen Y, Zhang F, Tsai Y, Yang X, Yang L, Duan S, Wang X, Keng P, Lee SO (2015) IL-6 signaling promotes DNA repair and prevents apoptosis in $\mathrm{CD} 133+$ stem-like cells of lung cancer after radiation. Radiat Oncol 10: 227.

Deorukhkar A, Krishnan S (2010) Targeting inflammatory pathways for tumour radiosensitization. Biochem Pharmacol 80(12): 1904-1914.

Diehn M, Cho RW, Lobo NA, Kalisky T, Dorie MJ, Kulp AN, Qian D, Lam JS, Ailles LE, Wong M, Joshua B, Kaplan MJ, Wapnir I, Dirbas FM, Somlo G, Garberoglio C, Paz B, Shen J, Lau SK, Quake SR, Brown JM, Weissman IL, Clarke MF (2009) Association of reactive oxygen species levels and radioresistance in cancer stem cells. Nature 458(7239): $780-783$.

Dubost JJ, Rolhion C, Tchirkov A, Bertrand S, Chassagne J, Dosgilbert A, Verrelle P (2002) Interleukin-6-producing cells in a human glioblastoma cell line are not affected by ionizing radiation. I Neuro-oncol 56(1): 29-34.

Fisher CJ, Goswami PC (2008) Mitochondria-targeted antioxidant enzyme activity regulates radioresistance in human pancreatic cancer cells. Cancer Biol Ther 7(8): 1271-1279.

Fisher DT, Appenheimer MM, Evans SS (2014) The two faces of IL-6 in the tumour microenvironment. Semin Immunol 26(1): 38-47.

Guo Y, Xu F, Lu T, Duan Z, Zhang Z (2012) Interleukin-6 signaling pathway in targeted therapy for cancer. Cancer Treat Rev 38(7): 904-910.

Hodge DR, Hurt EM, Farrar WL (2005) The role of IL-6 and STAT3 in inflammation and cancer. Eur J Cancer 41(16): 2502-2512.

Ichimura Y, Waguri S, Sou YS, Kageyama S, Hasegawa J, Ishimura R, Saito T, Yang Y, Kouno T, Fukutomi T, Hoshii T, Hirao A, Takagi K, Mizushima T, Motohashi H, Lee MS, Yoshimori T, Tanaka K, Yamamoto M, Komatsu M (2013) Phosphorylation of p62 activates the Keap1-Nrf2 pathway during selective autophagy. Mol Cell 51(5): 618-631.

Komatsu M, Kurokawa H, Waguri S, Taguchi K, Kobayashi A, Ichimura Y, Sou YS, Ueno I, Sakamoto A, Tong KI, Kim M, Nishito Y, Iemura S, Natsume T, Ueno T, Kominami E, Motohashi H, Tanaka K, Yamamoto M (2010) The selective autophagy substrate p62 activates the stress responsive transcription factor $\mathrm{Nrf} 2$ through inactivation of Keap1. Nat Cell Biol 12(3): 213-223.

Komohara Y, Jinushi M, Takeya M (2014) Clinical significance of macrophage heterogeneity in human malignant tumours. Cancer Sci 105(1): 1-8.

Kuwahara Y, Li L, Baba T, Nakagawa H, Shimura T, Yamamoto Y, Ohkubo Y, Fukumoto M (2009) Clinically relevant radioresistant cells efficiently repair DNA double-strand breaks induced by X-rays. Cancer Sci 100(4): 747-752.

Kuwahara Y, Mori M, Oikawa T, Shimura T, Ohtake Y, Mori S, Ohkubo Y, Fukumoto M (2010) The modified high-density survival assay is the useful tool to predict the effectiveness of fractionated radiation exposure. J Radiat Res 51(3): 297-302.

Lawenda BD, Kelly KM, Ladas EJ, Sagar SM, Vickers A, Blumberg JB (2008) Should supplemental antioxidant administration be avoided during chemotherapy and radiation therapy? J Natl Cancer Inst 100(11): 773-783.

Lee S, Lim MJ, Kim MH, Yu CH, Yun YS, Ahn J, Song JY (2012) An effective strategy for increasing the radiosensitivity of Human lung Cancer cells by blocking Nrf2-dependent antioxidant responses. Free Radic Biol Med 53(4): 807-816.

Liu X, Sun C, Liu B, Jin X, Li P, Zheng X, Zhao T, Li F, Li Q (2016) Genistein mediates the selective radiosensitizing effect in NSCLC A549 cells via inhibiting methylation of the keap1 gene promoter region. Oncotarget 7(19): 27267-27279.

Masuda M, Wakasaki T, Suzui M, Toh S, Joe AK, Weinstein IB (2010) Stat3 orchestrates tumour development and progression: the Achilles' heel of head and neck cancers? Curr Cancer Drug Targets 10(1): 117-126.

Matsuoka Y, Yoshida R, Nakayama H, Nagata M, Hirosue A, Tanaka T, Kawahara K, Nakagawa Y, Sakata J, Arita H, Hiraki A, Shinohara M (2015) The tumour stromal features are associated with resistance to 5 -FU-based chemoradiotherapy and a poor prognosis in patients with oral squamous cell carcinoma. APMIS 123(3): 205-214.

McDonald JT, Kim K, Norris AJ, Vlashi E, Phillips TM, Lagadec C, Della Donna L, Ratikan J, Szelag H, Hlatky L, McBride WH (2010) Ionizing radiation activates the Nrf2 antioxidant response. Cancer Res 70(21): 8886-8895.

Multhoff G, Radons J (2012) Radiation, inflammation, and immune responses in cancer. Front Oncol 2: 58.

Na HK, Kim EH, Jung JH, Lee HH, Hyun JW, Surh YJ (2008) (-)-Epigallocatechin gallate induces Nrf2-mediated antioxidant enzyme expression via activation of PI3K and ERK in human mammary epithelial cells. Arch Biochem Biophys 476(2): 171-177.

Nagata M, Nakayama H, Tanaka T, Yoshida R, Yoshitake Y, Fukuma D, Kawahara K, Nakagawa Y, Ota K, Hiraki A, Shinohara M (2011) Overexpression of cIAP2 contributes to 5-FU resistance and a poor prognosis in oral squamous cell carcinoma. Br J Cancer 105(9): $1322-1330$

Nakashima H, Matsuoka Y, Yoshida R, Nagata M, Hirosue A, Kawahara K, Sakata J, Arita H, Hiraki A, Nakayama H (2016) Pre-treatment neutrophil to lymphocyte ratio predicts the chemoradiotherapy outcome and survival in patients with oral squamous cell carcinoma: a retrospective study. BMC Cancer 16(1): 41 . 
Shinriki S, Jono H, Ota K, Ueda M, Kudo M, Ota T, Oike Y, Endo M, Ibusuki M, Hiraki A, Nakayama H, Yoshitake Y, Shinohara M, Ando Y (2009) Humanized anti-interleukin-6 receptor antibody suppresses tumour angiogenesis and in vivo growth of human oral squamous cell carcinoma. Clin Cancer Res 15(17): 5426-5434.

Shinriki S, Jono H, Ueda M, Ota K, Ota T, Sueyoshi T, Oike Y, Ibusuki M, Hiraki A, Nakayama H, Shinohara M, Ando Y (2011) Interleukin-6 signalling regulates vascular endothelial growth factor-C synthesis and lymphangiogenesis in human oral squamous cell carcinoma. J Pathol 225(1): 142-150.

Taguchi K, Motohashi H, Yamamoto M (2011) Molecular mechanisms of the Keap1-Nrf2 pathway in stress response and cancer evolution. Genes Cells 16(2): 123-140.

Taneja N, Davis M, Choy JS, Beckett MA, Singh R, Kron SJ, Weichselbaum RR (2004) Histone H2AX phosphorylation as a predictor of radiosensitivity and target for radiotherapy. J Biol Chem 279(3): 2273-2280.

Wu CT, Chen MF, Chen WC, Hsieh CC (2013) The role of IL-6 in the radiation response of prostate cancer. Radiat Oncol 8: 159.

Yoshida R, Nakayama H, Nagata M, Hirosue A, Tanaka T, Kawahara K, Nakagawa Y, Matsuoka Y, Sakata J, Arita H, Hiraki A, Shinohara M, Ito T (2014) Overexpression of nucleostemin contributes to an advanced malignant phenotype and a poor prognosis in oral squamous cell carcinoma. Br J Cancer 111(12): 2308-2315.
Yu H, Kortylewski M, Pardoll D (2007) Crosstalk between cancer and immune cells: role of STAT3 in the tumour microenvironment. Nat Rev Immunol 7(1): 41-51.

Zhang P, Singh A, Yegnasubramanian S, Esopi D, Kombairaju P, Bodas M, Wu H, Bova SG, Biswal S (2010) Loss of Kelch-like ECH-associated protein 1 function in prostate cancer cells causes chemoresistance and radioresistance and promotes tumour growth. Mol Cancer Ther 9(2): 336-346.

Zhang Y, Martin SG (2014) Redox proteins and radiotherapy. Clin Oncol (R Coll Radiol) 26(5): 289-300.

Zhao Q, Mao A, Yan J, Sun C, Di C, Zhou X, Li H, Guo R, Zhang H (2016) Downregulation of Nrf2 promotes radiation-induced apoptosis through Nrf2 mediated Notch signaling in non-small cell lung cancer cells. Int $J$ Oncol 48(2): 765-773.

Zhou S, Ye W, Shao Q, Zhang M, Liang J (2013) Nrf2 is a potential therapeutic target in radioresistance in human cancer. Crit Rev Oncol Hematol 88(3): 706-715.

This work is published under the standard license to publish agreement. After 12 months the work will become freely available and the license terms will switch to a Creative Commons AttributionNonCommercial-Share Alike 4.0 Unported License.

Supplementary Information accompanies this paper on British Journal of Cancer website (http://www.nature.com/bjc) 